\title{
Baroque
}

\section{Communication de Pierre Costabel}

\section{Pierre Costabel}

\section{OpenEdition}

\section{Journals}

Édition électronique

URL : http://journals.openedition.org/baroque/517

DOI : 10.4000/baroque. 517

ISSN : 2261-639X

\section{Éditeur :}

Centre de recherches historiques - EHESS, Éditions Cocagne

\section{Édition imprimée}

Date de publication : 1 mai 1980

ISSN : 0067-4222

\section{Référence électronique}

Pierre Costabel, «Communication de Pierre Costabel », Baroque [En ligne], 09-10 | 1980, mis en ligne le 15 mai 2013, consulté le 20 avril 2019. URL : http://journals.openedition.org/baroque/517 ; DOI $10.4000 /$ baroque. 517

Ce document a été généré automatiquement le 20 avril 2019

(c) Tous droits réservés 


\title{
Communication de Pierre Costabel
}

\author{
Pierre Costabel
}

\section{Intervention}

1 Le thème sur lequel je dois présenter quelques réflexions est celui du changement dans la conception du monde à la fin du XVI ${ }^{\mathrm{e}}$ siècle et au début du XVII ${ }^{\mathrm{e}}$ siècle. Le changement en question appartient à la panoplie des grandes affirmations sur lesquelles se construit notre univers mental, et nous l'admettons en général sans réfléchir au présupposé qui se trouve engagé. À savoir que nous avons le moyen de parler avec une exactitude suffisante de la "conception du monde ", et pour une époque très éloignée, tout de même, de la nôtre. Si les œuvres écrites nous permettent à une époque donnée d'avoir un accès relativement sûr aux idées généralement reçues chez les contemporains, il faut prendre garde. L'information correspondante n'est que partielle et un exemple me permettra tout de suite d'illustrer ce que je veux dire.

2 Dans la collégiale de Champeaux, près de Melun, on peut voir encore aujourd'hui, sur les « miséricordes » des stalles, d'admirables sculptures sur bois dues à l'art et à la verve d'un artisan parisien. Je dis la verve, car comment désigner autrement ce qui a présidé à la gravure de scènes cocasses de la vie quotidienne dans un ménage, ou de la manière dont le bon peuple fidèle subit les sermons de ses curés. Mais parmi les sujets d'inspiration populaire se trouve traité quelque chose que les rédacteurs du guide de visite n'ont pas compris. Sur le fond d'un ciel étoilé deux personnages encadrent à droite et à gauche un globe central surmonté d'une croix. Il s'agit évidemment de la Terre. L'un des personnages fait un geste d'arrière en avant, geste de lancement, tandis que l'autre fait aussi manifestement un geste de transmission de rotation. Aucun doute n'est donc possible. L'artisan, dont la facture de livraison date de 1525, avait l'idée du double mouvement de la Terre, et ceci dix-huit ans avant la publication du De Revolutionibus de Copernic (1543). Ce petit exemple suffit, me semble-t-il, à nous rendre prudent quant à notre appréciation de ce qui est rigoureusement neuf à une époque donnée. 
3 Naturellement tel ou tel d'entre nous pourrait m'objecter que Copernic a eu encore à souffrir la caricature vulgaire dont il fut l'objet au Carnaval de l'actuel Elblag. C'est-à-dire que s'il y avait à Paris un artisan tout disposé à recevoir ses idées, les paysans de Warmie étaient, au contraire, tout prêts à le ridiculiser, ou davantage. Sans doute, néanmoins l'artisan parisien a pour moi une grande importance en ce sens qu'il est le témoin certain de l'existence d'un terrain favorable à des idées nouvelles et qu'il postule, par sa nature technicienne et populaire, des cheminements souterrains antérieurs. Nous savons si peu de choses sur le développement de la cosmologie $a u \mathrm{Xv}^{\mathrm{e}}$ siècle, et sur la coupure très nette à cette époque entre les auteurs de livres et les praticiens de l'astronomie ! Je crois que ces derniers, plus proches du peuple, avaient en définitive beaucoup plus d'influence que nous ne pensons pour la diffusion d'idées refusées par les doctes.

4 Le cas Copernic n'a pas d'ailleurs fini de nous instruire. À travers l'énorme travail de traduction entrepris à l'occasion du cinquième centenaire de la naissance du promoteur d'une astronomie nouvelle, l'équipe qui est actuellement à l'œuvre constate qu'à part la première partie du De Revolutionibus orbium coelestium, très générale, la haute technicité de tout le reste, jointe à une rédaction latine fort peu claire, devait rendre le livre inaccessible à la grande majorité des « doctes ». Et si Copernic se méfiait de ceux-ci, il ne s'inquiétait pas moins - comme en témoigne le récit que Rhéticus nous a conservé d'un entretien entre amis, à Lubawa - des réactions du "sens commun " populaire. C'est-àdire qu'en définitive Copernic avait parfaitement conscience de ce que le milieu susceptible de l'entendre était très réduit, qu'il s'agisse de doctes ou de non doctes, et c'est là sans doute ce qui caractérise le plus véritablement la situation au milieu $\mathrm{du} \mathrm{XvI}^{\mathrm{e}}$ siècle.

5 Reprenant l'expression que j'employais en commençant et pour conclure cette introduction, je dirai que nous devons nous efforcer de rendre moins partielle notre information des mouvements d'idées au $\mathrm{XV}^{\mathrm{e}}$ siècle et d'intégrer à cette information tout ce qui provient d'ailleurs que de la littérature. Ceci afin d'avoir une notion plus exacte de la diversité sociale des individus porteurs de nouveauté, afin aussi de cesser de privilégier les débats proprement philosophiques au détriment de l'évolution des mentalités dans des milieux plus humbles et plus perméables à la réalité technique. Mais qu'il est quasi certain que Copernic avait raison de pratiquer tant de précautions dans la publication de son œuvre, et qu'à l'époque où il achevait sa rédaction, l'effectif des esprits susceptibles de la recevoir était encore trop faible. Que nul ne se scandalise de l'usage que je fais d'un terme emprunté à la langue militaire ; ce terme est adéquat à mon avis.

6 Il est clair que c'est cette situation qui s'est trouvée complètement retournée au début du $\mathrm{XVII}^{\mathrm{e}}$ siècle. Lorsque parait le décret romain de 1616 autorisant la lecture de Copernic pour son hypothèse mathématique de description des apparences, mais interdisant de prendre cette hypothèse comme expression possible sur le plan de la vérité physique, il s'agissait en fait de la reprise pure et simple de précautions suggérées par la préface même du De Revolutionibus (par Osiander) et cela caractérise déjà le chemin parcouru depuis 1543. Officialiser, trois quarts de siècle après, la prudence proposée par les éditeurs de Copernic, c'est sans doute répondre bien tard à leur captatio benevolentiae, mais est surtout manifester que l'heure n'est plus au silence. C'est-à-dire que la diffusion d'idées nouvelles, touchant la cosmologie, en est venue à un degré suffisant pour que l'autorité ecclésiastique soit sollicitée de donner son avis. 
7 Et il est toujours dangereux pour les autorités, quelles qu'elles soient, d'intervenir en matière de mouvement d'idées. Le décret de 1616 a fait connaitre Copernic au-delà des milieux où on entendait déjà parler de lui. Je considère comme un excellent témoignage à cet égard la mention faite par le Cardinal de Bérulle en 1619, dans un ouvrage de spiritualité sur les Grandeurs de Jésus. Déclarer que si le soleil, centre des mouvements planétaires, n'est pas une idée susceptible d'être soutenue en matière d'astronomie, elle est néanmoins fort utile sur le plan mystique pour fournir une image du rôle de Jésus dans la création, c'est bien souligner la puissance de suggestion de la cosmologie nouvelle.

Je m'en tiendrai là. J'ai simplement voulu marquer comment il me semble qu'il est possible de prendre une position méthodologique plus avisée quand il s'agit de parler de la conception du monde d'un temps donné.

Mais je crois utile d'ajouter que nous devons être attentifs, en ce qui concerne l'époque que nous évoquons ici, à la manière dont les livres se «fabriquaient » et à ce qui servait en fait de véhicule aux idées. Le cas de Mersenne: le secrétaire de l'Europe savante, permet d'illustrer les deux volets de cette situation historique. Mersenne avait une correspondance considérable et ne gardait pour lui aucun secret. Mais il ne malmenait pas seulement les susceptibilités de ses correspondants. Il écrivait beaucoup à tous les points de vue et avait une manière éprouvante de faire des livres. Il passait chez son imprimeur pour faire des corrections sur place et substituer des « cartons » aux pages qui lui paraissaient à modifier. Au point qu'il n'y a pas deux exemplaires de ses ouvrages dont on puisse dire qu'ils soient parfaitement identiques. C'est un cas extrême, mais la "bibliographie matérielle", d'origine anglo-saxone et que nous pratiquons à l'Équipe Descartes, montre combien il faut se méfier dans de nombreux autres cas. Sous la même date d'édition, $\mathrm{au} \mathrm{XVII}^{\mathrm{e}}$ siècle, on a très souvent des "émissions" différentes, et différentes sur un nombre de points restreint mais correspondant précisément aux questions débattues et délicates. Newton, par exemple, ayant d'abord écrit dans l'addition à la traduction latine de son Optique que l'espace est "sensorium Dei ", s'est repenti devant les protestations de Leibniz et a introduit un carton avec «tanquam sensorium Dei ». C'est cette version, habilement modulée dans le style scolastique, qui était seule connue jusqu'à ce que l'on retrouve, il y a peu, un exemplaire de la première émission. Motivée par le fait que la protestation de Leibniz était connue par sa correspondance avec Clarke, la recherche a donc abouti à laver le grand adversaire de Newton du soupçon de mauvaise foi, et à propos d'une question importante au point de vue philosophique. L'exemple vaut aussi pour illustrer l'intérêt des correspondances. Cet intérêt, signifié de manière singulière par la publication de l'énorme dossier de Mersenne, n'a cessé depuis vingt ans de s'accroître et de porter des fruits à propos de nombreux auteurs.

10 Si une presse périodique savante a commencé à voir le jour dans la seconde moitié du XVII e siècle, il reste que jusqu'à la fin de ce siècle il faut tenir le plus grand compte de la circulation des idées par le moyen de copies et de lettres. C'est-à-dire en définitive qu'il faut avoir le sens du bouillonnement des idées dont la littérature ne donne jamais qu'une superstructure. Par rapport au thème de la conception du monde et à la préoccupation qui nous réunit ici - celle du Baroque - il est clair que la conscience de cette situation est très importante.

11 Il faut cependant que j'en vienne à la question même que vous m'avez posée. Comment indiquer brièvement ce qui caractérise, pour l'historien des sciences, un changement dans la conception du monde du $\mathrm{XVI}^{\mathrm{e}}$ au XVII ${ }^{\mathrm{e}}$ siècle ? Il me semble que le colloque tenu à 
Royaumont en 1957 (Actes publiés chez Hermann dans la collection Histoire de la Pensée) définit à cet égard les bonnes perspectives. Le changement n'est pas dans cette «Révolution copernicienne » telle qu'Auguste Comte l'a célébrée, c'est-à-dire dans une cosmologie renouvelée par la substitution du Soleil à la Terre comme centre des mouvements planétaires.

Sans doute ne doit-on pas négliger le fait d'une cosmologie qui supprime le privilège détenu jusque-là par la Terre, privilège curieusement conjugué d'ailleurs avec la localisation du corruptible, mais il est certain que la cosmologie nouvelle n'est que l'une des composantes des perspectives qui s'ouvrent à l'esprit de l'homme au milieu du XVI siècle. Cette cosmologie restait affaire spéculative et savante tandis que l'impact de la découverte du Nouveau Monde était autrement positif et sensible au plus grand nombre. La preuve était faite d'un certain nombre de chimères comme les hommes ayant leur tête dans la poitrine, la preuve était faite de l'extension des terres habitées et de l'homogénéité fondamentale - malgré des différences - du monde vivant. Et il est remarquable que c'est cette expérience-là qui enrichit la cosmologie, comme on peut le constater chez un Giordano Bruno, et non l'inverse. Autrement dit, le changement de conception qui nous intéresse a commencé essentiellement par un agrandissement de la connaissance du cadre terrestre et lorsqu'un Galilée étend, à travers les observations que permet la lunette, l'agrandissement aux cieux, il ne faut que proposer la première tentative positive - et tardive - pour vérifier le bien-fondé d'une homogénéité générale de la Création postulée depuis longtemps.

Qu'il faille prendre ses distances par rapport à l'interprétation d'A. Comte apparait de plus en plus, aujourd'hui, nécessaire. Dès 1630, Mersenne déclarait dans sa correspondance que la notion de centre du monde est à éliminer parce que l'univers est infini. Et c'est ce qui a suggéré à Alexandre Koyré le titre même d'un de ses ouvrages : «Du monde clos à l'univers infini ». C'est là la bonne formule. Le changement de la conception du monde, à l'époque considérée, n'est pas signifié par une cosmologie qui substitue un centre à un autre, mais par un agrandissement au-delà de toute limite. Agrandissement besogneusement commencé sur Terre et dans des domaines de connaissances très variés, et non moins besogneusement poursuivi dans les cieux par les promoteurs d'une astronomia restaurata.

Ce sont là des notes très générales, mais dont vous pouvez aisément trouver l'analyse dans des ouvrages récents comme ceux que j'ai cités. Il me paraît plus utile de souligner quelques éléments qui sont d'ordinaire considérés comme sans rapport avec la question. Par exemple, le fait que le seul écrit que Copernic ait publié de son vivant ait été un opuscule sur la nécessité d'un code monétaire européen. Cette publication illustre la lutte que Copernic en tant que responsable des finances du diocèse de Wannie, eut à mener contre les Chevaliers Teutoniques, lesquels pratiquaient effrontément sur les monnaies d'argent des opérations frauduleuses. Opérations perturbatrices des échanges commerciaux et dont le petit peuple avait à souffrir plus que tous autres. On aurait tort de croire que ceci n'intéresse guère notre thème. En se penchant sur la nécessité d'un code monétaire qui serait admis par tous les pays, jusqu'au Portugal, Copernic manifestait qu'il n'était plus possible, en son temps, de vivre en limitant sa vision aux frontières de son pays.

Voici, d'ailleurs, dans le même ordre d'idées, quelque chose de plus directement lié à notre thème. Lorsqu'on parle de la science à la fin du XVI ${ }^{e}$ siècle, on omet en général de dire que la diversité des unités de mesure constituait une difficulté considérable. 
L'apparition entre 1620 et 1640 de plusieurs traités concernant la correspondance des mesures depuis l'Antiquité et selon les pays a donc été significative d'une prise de conscience. Prise de conscience de ce que la communication du savoir et la constitution d'une science universelle imposent l'établissement d'un code des langages - c'est évidemment là la première évidence - mais prise de conscience qui est elle-même la conséquence de l'agrandissement des perspectives mentales auquel nous avons fait allusion plus haut.

Il est d'ailleurs intéressant de constater que se manifeste en même temps le souci de reconnaitre ce qui relève de l'universel, indépendamment des mesures, et se trouve donc vérifié en tout lieu. Lorsque Mersenne fait connaitre au milieu français, en 1638-1639, le contenu du manuscrit des Discorsi de Galilée qu'il a eu entre les mains avant publication, il prend soin de bien fixer la correspondance des unités de longueur italiennes et françaises, mais il ne dit rien des unités de poids parce que la loi de la chute des corps n'en dépend pas. Et lorsqu'il spécule, à la suite, sur l'oscillation du pendule, il est très clair qu'il conçoit par là le moyen de mesurer le temps d'une manière qui soit indépendante du lieu de l'expérience. À travers le problème du code des mesures, nécessaire pour la constitution d'un savoir communicable au-dessus des frontières, s'affirme donc l'idée des lois physiques valables quelle que soit la localisation terrestre et avant leur traduction numérique dans un système particulier. Et c'est bien là à la fois une conséquence ultime de l'agrandissement de l'univers mental des amateurs de science et un aspect important du changement acquis dans la conception du monde.

Bien entendu, cela ne veut pas dire que l'universel ne se trouvait pas en bute à des débats contradictoires. Puisque j'ai évoqué la mesure du temps (chronométrie), il me faut souligner que les choses n'allaient pas si bien en matière de chronologie. La réforme du calendrier - dite réforme grégorienne, 1582 - témoigne de ce que les milieux romains avaient au moins retenu de l'œuvre de Copernic la nécessité de corrections dans la définition de l'année et la répartition des années bissextiles. Mais on sait combien cette réforme fut combattue par les pays protestants, pour des raisons politiques qui ne devaient rien à la science. Et l'opposition que le mathématicien bien connu, François Viète, fit à la réforme pour des raisons scientifiques, resta, elle, parfaitement sans écho.

Il est vrai que Viète n'adhérait pas à la cosmologie copernicienne et lui préférait celle de Tycho Brahe. Ce qui m'amène à dire que les débats contradictoires auxquels je viens de faire allusion ne sont pas toujours vulgaires.

En voici un exemple. Le 12 août 1654 était attendue une éclipse de soleil qui devait être visible dans toute l'Europe et l'attente avait été l'objet d'une campagne des astrologues parce que le soleil devait être en conjonction non seulement avec la lune, mais aussi avec Saturne l'astre maléfique. On assurait la fin du monde et la panique fut telle que dans toutes les confessions chrétiennes les prédicateurs furent mobilisés pour répondre à l'angoisse générale. Certains en profitèrent pour rendre plus efficace un appel à la pénitence, quelques-uns - comme les Lazaristes polonais qui firent consulter Gassendi par saint Vincent de Paul - cherchèrent à combattre la terreur par la raison. Une de nos collègues de Paris, Mme Labrousse, a fait une étude très complète de ce phénomène social et religieux, étude qui doit paraître chez Nijhoff dans la collection des Archives Internationales d'Histoire des idées sous le titre que je lui ai suggéré: "L'entrée de Saturne au Lion ». Car il y a évidemment un lien entre cette expression qui figure dans les Pensées de Pascal et les événements d'août 1654, comme il y a un lien entre ces 
événements et la fameuse nuit de feu du même Pascal le 23 novembre. L'argument de la raison en pareille affaire, celui de Gassendi comme celui qui est sous-jacent chez Pascal, était que la périodicité des phénomènes tels que conjonctions d'astres et éclipses interdit de leur donner un sens surnaturel. Mais cette périodicité, bien que plus aisée à concevoir, et à calculer dans le système copernicien, ne lui était pas cependant liée de manière absolue. L'exemple me parait donc assez significatif de ce que, en plein milieu du XVII siècle, la mentalité populaire offrait encore de prises à la propagande des charlatans de l'astrologie - au mépris de ce que la cosmologie ancienne était pourtant encore capable d'expliquer raisonnablement.

Mais si les doctes hésitaient - dans des circonstances aussi dramatiques - à proclamer l'avantage du système copernicien, qui les aidait en fait à mieux concevoir la solution des angoisses communes, c'est que la cosmologie nouvelle aboutissait, comme je l'ai dit plus haut, à l'idée d'un univers infini. D'où des problèmes philosophiques redoutables que l'on trouve sous-jacents chez les grands promoteurs de la science de la fin du siècle. Que ce soit chez un Huygens, qui s'en accommodait mal, ou chez Newton, qui apparemment s'y résignait mieux par quelques artifices de langage. Sur le plan de la représentation des phénomènes, les praticiens de l'observation astronomique ne trouvaient pas dans la cosmologie nouvelle un avantage suffisamment sûr du point de vue de la transformation de leurs calculs. C'est un point qui mériterait de plus amples explications, mais il suffit de le signaler pour confirmer, comme je le disais il y a un instant, que les résistances à la pénétration des idées nouvelles n'étaient pas vulgaires.

Que conclure, sinon ceci : loin de s'achever à la fin du XVII siècle dans la sérénité, le changement de la conception du monde amorcé au XVI poursuivait un chemin tortueux, ponctué de conflits et de tensions - certaines situées à un haut niveau intellectuel. Il me semble que c'est dans la mesure où les doctes ont vécu ces tensions que l'on peut rechercher en eux des témoins de l'atmosphère baroque, c'est-à-dire d'une atmosphère de désarroi non pleinement dominé par les sommets.

\section{Discussion}

La discussion qui s'engage, un peu à bâtons rompus, montre que les littéraires présents se sentent provoqués. Certains réclament mention de l'influence d'auteurs célèbres comme Du Bartas et Jean Bodin, témoins importants du bouillonnement des idées concernant la conception du monde et sur la toile de fond biblique. Qu'il s'agisse d'accéder à la Parole de Dieu créatrice, au Logos, avec Du Bartas - ou de prolonger dans l'histoire de l'humanité le mythe de la Tour de Babel, avec Jean Bodin. Et il est vrai que la diffusion de ces auteurs a été considérable : Florimond de Beaune, conseiller au Présidial de Blois, mathématicien distingué et disciple de Descartes, leur avait fait place dans sa bibliothèque.

D'autres interventions soulignent la floraison de la littérature relative aux mondes imaginaires, paraphrases de la Genèse dont l'Abbaye de Thélème semble avoir ouvert la série. On signale encore le rôle des spéculations numériques et de la Caballe (Postel) à travers lesquelles l'histoire du monde est perçue selon des «âges » où le devenir est en même temps dégradation. On évoque les poètes qui, comme Sponde, opposent la stabilité de l'amour à l'instabilité de l'Univers, ou comme les Pétrarquistes usant du mythe solaire dans une perspective dynamique. 
24 La preuve est faite que le thème abordé engage l'attention à des données extrêmement diverses et le dialogue entre littéraires et scientifiques. S'il est exact, comme l'a affirmé un intervenant, que la crise de la fin du XvI ${ }^{\mathrm{e}}$ siècle est surtout d'ordre moral, il reste tout de même que le développement des connaissances et le mouvement des idées scientifiques y tiennent une place que seule la collaboration intelligente des uns et des autres permettra de mieux mesurer.

25 - AUZIAS attire l'attention sur la différence entre «fait social total» et "société globale » : le premier concept désignant un universel concret, le point où convergent et se focalisent les diverses activités d'un groupe. Le second au contraire se réfère à l'instance ultime au sein de laquelle tous les universels concrets viennent se situer.

26 - Une réflexion se développe sur l'éventuelle unité des méthodes et la constitution d'équipes de travail. Pour J. KRYNEN chacun d'entre nous apporte des problématiques différentes et nous sommes ici pour les faire converger. Le carrefour nous renvoie sur des allées très divergentes.

27 - Marguerite SOULIÉ : Très peu de gens ont le Baroque pour champ de recherche. Je me sens personnellement très liée à cette recherche: elle n'en reste pas moins marginale pour moi. C'est le cas général dans l'Université française.

28 - CASTAN approuve le maintien des découpages traditionnels. La notion de Baroque, comme on l'a dit tout au long de ces trois jours, intervient seulement au niveau de la synthèse interdisciplinaire. Le va-et-vient entre l'analyse du spécialiste et la synthèse de civilisation, cette bi-polarité de la démarche doit être préservée comme essentielle. Anne Viguier préconise la constitution de groupes de travail. On demande avec J. KRYNEN la participation de linguistes. F. COURTÈS attend des linguistes qu'ils disent à quelle phase de la constitution des langues européennes correspond l'époque baroque.

29 - DRIJKONINGEN : Comme il a été dit hier, nous sommes au moment où un verbe nouveau prend la parole : la jonction du langage est le problème fondamental du Baroque. Peutêtre le cartésianisme apporte-t-il une théorie révolutionnaire du langage. Mais ne peuton faire l'hypothèse que ce soit le Baroque qui par sa pratique du langage en fait valoir l'ambiguïté et la puissance créatrice?

30 - Anne VIGUIER expose sa méthode d'analyse et de preuve par le moyen linguistique. Elle a étudié le mot « expérience » au XVI ${ }^{\mathrm{e}}$ siècle et suggère l'étude du mot « hauteur » chez les baroques, sur lequel un échange s'improvise.

31 - DUBOIS croit que la mythologie renaissante comporte une dominance du mythe de la terre et de la mère. Au Baroque la dominance serait du père et du soleil, d'où la recherche par le fils symbolique du père symbolique, l'envol, l'aspiration vers le ciel et les hauteurs. J. KRYNEN demande des preuves plus précises. J-C. BRUNON admet chez Hopil l'ascension et la symbolique du père, mais la dévotion mariale y est égale en importance.

32 - CASTAN et COURTÈS : les contextes sont plus importants que le fait. J-M. Auzias : quel rapport de l'étude ponctuelle avec le vécu, ce qui se transmet, la communication?

33 - À l'initiative de C. DUBOIS, une contestation approfondie est menée contre le terme de "structures mentales", inutilisable, invérifiable, à raisonner en toute rigueur. Autocritique généralisée. Sauf peut-être dans une perspective chomskyenne.

34 - Papin soulève le problème du roman baroque. N'existe-t-il donc pas un roman baroque ? On parle trop exclusivement de poésie et de théâtre. Don Quichotte, Simplicissimus et tant d'autres sont là pour montrer que le roman réaliste apparait en concomitance avec 
tout ce qu'on appelle baroque. Des études se poursuivent en Italie et en France (L'Astrée, Francien, Cyrano, etc.).

35 - Autres thèmes :

Le rapport du Baroque et du fantastique?

Idéologie et cadre formel?

\section{AUTEUR}

PIERRE COSTABEL

Directeur d'Études - École Pratique des Hautes Études 